\title{
Terpene Synthase Gene OtLIS Confers Wheat Resistance to Sitobion avenae by Regulating Linalool Emission
}

\author{
Yidi Zhan, ${ }^{\S}$ Lei Zhao, ${ }^{\S}$ Xiaojing Zhao, Jiahui Liu, Frederic Francis, and Yong Liu*
}

Cite This: https://doi.org/10.1021/acs.jafc.1c05978

Read Online

ACCESS

Wlll Metrics \& More

Article Recommendations

Supporting Information

ABSTRACT: Sitobion avenae (Fabricius) is a major insect pest of wheat worldwide that reduces crop yield and quality annually. Few germplasm resources with resistant genes to aphids have been identified and characterized. Here, octoploid Trititrigia, a species used in wheat distant hybridization breeding, was found to be repellent to $S$. avenae after 2 year field investigations and associated with physiological and behavioral assays. Linalool monoterpene was identified to accumulate dominantly in plants in response to $S$. avenae infestation. We cloned the resistance gene OtLIS by assembling the transcriptome of aphid-infested or healthy octoploid Trititrigia. Functional characterization analysis indicated that OtLIS encoded a terpene synthase and conferred resistance to $S$. avenae by linalool emission before and after aphid feeding. Our study suggests that the octoploid Trititrigia with the aphid-resistant gene OtLIS may have potential as a target resource for further breeding aphid-resistant wheat cultivars.

KEYWORDS: aphid resistance, gas chromatography-mass spectrometer, octoploid Trititrigia, RNA-seq, S-linalool synthase, volatile terpenoids

\section{INTRODUCTION}

Outbreaks of the grain aphid, Sitobion avenae (Fabricius), on the wheat (Triticum aestivum L.) filling stage in a temperate zone threaten wheat production and food safety. ${ }^{1-4} S$. avenae causes significant yield losses in cereals worldwide by phloem sap-sucking, excreting honeydew, and transmitting plant viruses. $^{5-8}$ Aphid control in cereal production necessitates routine insecticide use with undesirable environmental effects and an increase in pest insecticide resistance. ${ }^{9}$

Considerable efforts have been made in conventional breeding and genetically modified programs to breed for resistance against aphids in wheat. ${ }^{10-14}$ Nevertheless, the development of aphid-resistant wheat cultivars has not reached such success to suppress aphid abundance in the fields, as a result of the complexity of plant-aphid-natural enemy interactions. $^{10,11,13}$ Moreover, most wheat cultivars were found to be susceptible to aphid attack, and the resistance genes do not exist in the gene pool of modern wheat varieties. ${ }^{15-18}$ Breeders and growers are still struggling to find an efficient strategy for aphid control by continuously facing the aphid outbreaks in wheat production. ${ }^{16,19-21}$ Then, screening potential genes from wheat germplasm resources for performing crosses between different species, genera, higher-ranking taxa, or by genetic engineering may make it possible to transfer aphid-resistance genes to wheat.

During the long process of suffering from phytophagous insects, plants have evolved a variety of defense mechanisms, including constitutive and inducible defenses, which has cast a new light against aphids. ${ }^{22-25}$ Terpenes, a major class of volatile organic compounds (VOCs), generally play vital roles in repelling herbivores and attracting predators or parasitoid in plant-induced defense. $^{26-29}$
Volatile terpenoids are mainly synthesized through the mevalonate (MVA) pathway in the cytoplasmic/endoplasmic reticulum or the 2-c-methyl-d-erythritol 4-phosphate (MEP) pathway in the plastids. ${ }^{30-32}$ Geranyl diphosphate (GPP), farnesyl diphosphate (FPP), and geranylgeranyl diphosphate (GGPP) as acyclic substrate molecules were converted into mono-, sesqui-, and diterpene skeletons, respectively, by specialized terpene synthases (TPSs). ${ }^{33}$ Based on phylogenetics and functional studies, TPSs are commonly divided into eight subfamilies: TPS-a (sesquiterpene synthases), TPS-b (cyclic monoterpene and hemiterpene synthases), TPS-c (copalyl diphosphate synthases), TPS-d (gymnosperm-specific terpene synthases), TPS-e (ent-kaurene synthases), TPS-f (other diterpene synthases), TPS-g (acyclic monoterpenes), and TPS-h (specific to lycopod Selaginella moellendorffii). ${ }^{34,35}$ Among these, the number of TPS-g and TPS-b has been in vitro identified to catalyze GPP, FPP, and GGPP into monoterpenes, sesquiterpene, and diterpene, respectively.

Linalool is acyclic monoterpene alcohol produced by monoterpene synthase in many plants, which has a sweet floral fragrance. ${ }^{36}$ The gene synthesizing linalool has been characterized and isolated in many cash crops, including Camellia sinensis, Gossypium hirsutum, Citrus unshiu Marc, Cinnamomum osmophloeum, Vitis vinifera, and others. ${ }^{29,37-40}$ These results indicated that linalool synthases were able to convert the GPP into S-linalool and FPP into (E)-nerolidol.

Received: September 23, 2021

Revised: November 4, 2021

Accepted: November 4, 2021 
Several studies have shown that linalool emission plays important roles in plant defense mechanisms by repelling pests and deterring oviposition. For example, the transgenic Nicotiana tabacum L. plants overexpressing the cotton GhTPS12 gene, which produced a significantly higher level of (3S)-linalool, showed direct defense responses against Helicoverpa armigera female adults and Myzus persicae. ${ }^{29}$ Moreover, S-linalool is a key component with defense effects against Nilaparvata lugens Stål ${ }^{41}$ and is the most abundant VOC emitted from rice plants damaged by the fall armyworm Spodoptera frugiperda Smith \& Abbot larvae. This cue significantly attracts parasitic wasps. ${ }^{42}$ Hence, linalool may also have potential in the manipulation of plant defense focusing on biological control of herbivores through insectresistant cultivar breeding.

Thinopyrum intermedium (host) Barkworth \& Dewey, as a close relative of wheat, has many valuable attributes such as being multiflorous, resistance to pests and diseases, and tolerance to salt and drought. It is one of the most important wild resources that has been widely used in genetic improvement of wheat. ${ }^{43}$ The octoploid Trititrigia was selected from the common wheat and Th. intermedium (wheat-Th. intermedium partial amphiploid) cross. It could comprehend the superior traits of parents and act as a bridge parent for transferring superior genes of Th. intermedium grass to wheat. ${ }^{44}$ Based on 2 year field observations on aphid population growth over time and field experiments with the octoploid Trititrigiaresistant level to $S$. avenae during the wheat filling stage, we first hypothesized that some VOCs could be emitted from octoploid Trititrigia and deter $S$. avenae from the host plant. The deterring effects might be enhanced by aphid damages. Then, VOCs were collected and analyzed by gas chromatography associated to mass spectrometry (GC-MS) to compare the chemical profiles and investigate active compounds through olfactory tests. Second, we hypothesized that some terpene synthases might be involved in terpenoid biosynthesis. Therefore, a de novo comparative transcriptomic analysis between intact and aphid-damaged octoploid Trititrigia was performed. Third, the biosynthesis process was hypothesized to be mediated by terpene synthase genes. The terpene synthase gene of functional volatile terpenoid was cloned and the enzyme was expressed in vitro prior to functional characteristic assessment. These data not only strengthen new insights into plant defense responses to $S$. avenae but also provide a better understanding of the mechanism underlying constitutive and induced defense response of cereal crops to aphids, especially providing applicable candidate resistance genes for breeding wheat cultivars.

\section{MATERIALS AND METHODS}

Insects and Plants. The $S$. avenae aphid colony was reared on wheat seedlings (Lumai 21 variety) in a controlled condition chamber $\left(21^{\circ} \mathrm{C}, 16: 8 \mathrm{~h}\right.$ photoperiod and $70 \%$ relative humidity), with new wheat seedlings provided once a week. Octoploid Trititrigia was provided by Dr. Xingfeng Li from the National Wheat Improvement Center of China. We declared that the collection of the plant materials complies with institutional, national, or international guidelines.

Resistance Assessment and Treatment Design in Fields. Wheat, octoploid Trititrigia, and triticale (intergeneric cross between wheat and rye) were sown in the early October, 2015 and 2016 in fields $\left(35^{\circ} 38^{\prime} \mathrm{N}, 116^{\circ} 02^{\prime} \mathrm{E}\right)$ to test their resistance to $S$. avanae according to the Rule for Resistance Evaluation of Wheat to Aphids of China (NY/T 1443.7-2007). Winged and wingless aphids were recorded on 5 weekly sampling dates over aphid damage periods (from 10 April to 25 May). Common wheat Lumai 21 was considered as control. In 2017, six plots $\left(1 \mathrm{~m}^{2}\right.$ each) sowed with octoploid Trititrigia were set at the same location. Plots were individually covered by an insect-proof screen. Three to five plants in each plot were treated by introducing 100 wingless adults of $S$. avanae onto the heads of each plant at the flowering stage with triplicates. The other three plots without aphid infestation served as a control. Both intact and damaged wheat heads were sampled after $72 \mathrm{~h}$.

Volatile Collection and Gas Chromatography-Mass Spectrometer (GC-MS) Analysis. Sampling heads infected by aphids for $72 \mathrm{~h}$ were collected in the field. The air entrainment (dynamic headspace collection) was carried out following standard procedures using the flowering heads of octoploid Trititrigia. For each headspace collection, a head was enclosed in a gas sampling bag (polytetrafluoroethene, $2 \mathrm{~L}$, E-Switch) opened at the bottom, and with two collection ports at both sides (one for the inlet of air and the other for the outlet). The bottom was closed without pressure around the plant stem by a strip. Air was purified by a passage through an activated charcoal filter and was pumped into the vessel through the inlet port at $600 \mathrm{~mL} \cdot \mathrm{min}^{-1}$. The air was drawn out at $400 \mathrm{~mL} \cdot \mathrm{min}^{-1}$ through 50 mg Porapak Q (Alltech Associates, Carnforth, Lancashire, U.K.) in a 5 $\mathrm{mm}$ diameter Tenax glass tube. The difference in the flow rate created a slight positive pressure within the collection bag to prevent unfiltered air from entering into the system. All connections were made with a poly(tetrafluoroethylene) (PTFE) tubing with brass ferrules and fittings were then closed with PITE tape. Porapak Q-filled tubes were conditioned before use by washing with $n$-hexane $(1 \mathrm{~mL})$ and heating inside an oven $\left(150^{\circ} \mathrm{C}\right)$ under a stream of nitrogen for a minimum of $2 \mathrm{~h}$. Volatiles were collected on Porapak Q tubes inserted into the collection port on the top of the vessel and were subsequently eluted with $500 \mu \mathrm{L}$ of fresh hexane. Air entrainment samples were analyzed by an Agilent $7890 \mathrm{~A}$ gas chromatograph coupled with a $7000 \mathrm{D}$ mass spectrometer (Agilent Technologies, Palo Alto, CA). For air entrainment samples, aliquots of samples $(1 \mu \mathrm{L})$ were analyzed using a DB5-MS column $(30 \mathrm{~m} \times 0.25 \mathrm{~mm}$ ID $\times 0.25 \mu \mathrm{m}$ film thickness; Agilent Technologies) with a split/splitless injector (splitless mode, $220{ }^{\circ} \mathrm{C}$ ). Helium was used as a carrier gas at 1 $\mathrm{mL} \cdot \mathrm{min}^{-1}$; the oven temperature was programmed to increase from 50 to $60{ }^{\circ} \mathrm{C}$ (5 min hold) with a rate of $5{ }^{\circ} \mathrm{C} \mathrm{min}^{-1}$ and then increased to $250{ }^{\circ} \mathrm{C}$ with a rate of $10{ }^{\circ} \mathrm{C} \mathrm{min}^{-1}$ ( $5 \mathrm{~min}$ hold). The transfer line temperature was $250^{\circ} \mathrm{C}$ and the ion source temperature was $250{ }^{\circ} \mathrm{C}$. Ionization was performed by electron impact $(70 \mathrm{eV})$, and the scanned range was between $\mathrm{m} / z 50$ and 650 . Volatiles were identified by comparison of their GC retention times and mass spectra with authentic references.

Physiological and Behavioral Assays. The electroantennography (EAG) tests were performed to determine the antennal responses of $S$. avenae to $S$-linalool as previously described. ${ }^{45,46}$ Antennae were excised from the base and attached immediately with the signal gel (Parker Laboratories, INC) between two electrodes. The base and tip of antennae were connected with the reference electrode and the recording electrode, respectively. For EAG, $10 \mu \mathrm{L}$ of $S$-linalool dissolved in $n$-hexane (Kaitong, Tianjin, 98.0\%) at the concentrations of $0.01,0.1,1,10$, and $100 \mu \mathrm{g} \cdot \mu \mathrm{L}^{-1}$ was injected into the filter paper $(1 \mathrm{~cm} \times 1 \mathrm{~cm})$ and placed in a Pasteur pipette. Five dilutions of the compound from the lowest to the highest concentrations were tested. Only one concentration was detected for each antenna, and the trials with each EAG preparation were repeated three times. Six antennae were measured for each concentration. N-hexane was used as a control before and after each antennae test. The end of the Pasteur sample tube was connected with the stimulus controller (model CS-55, Syntech, The Netherlands). An antenna was placed $1 \mathrm{~cm}$ from the end of the airflow tube with a $10 \mathrm{~mL} \cdot \mathrm{s}^{-1}$ air rate and stimulus duration of $0.5 \mathrm{~s}$ with a $30 \mathrm{~s}$ interval. Pressing the foot pedal after baseline balanced was followed by EAG response measurement. The Pasteur pipette and the filter paper were replaced each time when the compound was changed. The relative value of antennal potential response $(\mathrm{mV})=$ Response to odor stimulation $(\mathrm{mV})$ - Average value of response 
before and after control test $(\mathrm{mV})$. One-way ANOVA and Tukey's test $(P<0.05)$ were used to analyze whether the EAG reaction values have significant differences.

The behavioral assays were performed using a four-arm olfactometer in a controlled room $\left(21 \pm 1{ }^{\circ} \mathrm{C}\right.$ and $70 \pm 5 \%$ relative humidity). The olfactometer was built in Plexiglas with a $10.5 \mathrm{~cm}$ walking area and connected to a vacuum pump to extract air. The flow rate of each arm was $150 \mathrm{~mL} \cdot \mathrm{min}^{-1}$. Volatile chemical was dissolved in $\mathrm{n}$-hexane (Kaitong, Tianjin, $98.0 \%$ ) at five concentrations (0.01, $0.1,1,10$, and $100 \mu \mathrm{g} \cdot \mu \mathrm{L}^{-1}$ ) and $n$-hexane was used as a control. One of the four source bottles contained a $1 \mathrm{~cm}^{2}$ piece of filter paper soaked with $20 \mu \mathrm{L}$ of the tested solution, while the other three bottles contained filter paper soaked with $20 \mu \mathrm{L}$ of $n$-hexane as controls. Each aphid was placed at the center of the exposure chamber and observed for $15 \mathrm{~min}$ to record the time spent in each olfactometer area. A total of 20 replicates were performed for each concentration, and each aphid was tested only once. For every two observations, the olfactometer was rotated by $90^{\circ}$ to avoid directional bias. Between each treatment, the system was cleaned with pure ethanol and rinsed with distilled water. Test data were expressed as "mean \pm standard deviation" and IBM SPSS 20.0 was used for corresponding analysis and test. The nonparametric test (Wilcoxon signed rank-sum test, and the test quantity was expressed by W) for the two correlated samples was performed for the aphid residence time in the treatment arm and $1 / 3$ of the residence time in the control arm.

Transcriptome Analysis of Octoploid Trititrigia Before and After Aphid Infestation. Transcriptome analysis was performed both on intact and aphid-infected wheat heads. ${ }^{47}$ Total RNA was extracted from three biological replicates of the flowering head using a Trizol reagent (Invitrogen) according to the manufacturer's instructions and then treated with RNase-free DNase I (Invitrogen). Each biological replicate contained a single flowering head. The quality and concentration of the total RNA were determined using a NanoPhotometer spectrophotometer (Implen, CA) and the Qubit RNA Assay Kit with a Qubit 2.0 Fluorometer (Life Technologies, CA), respectively. The RNA integrity was confirmed using an Agilent 2100 Bio-Analyzer (Agilent Technologies, CA). RNA samples with RIN $\geq 7$ and $28 \mathrm{~S} / 18 \mathrm{~S}$ ratio $\geq 1.5: 1$, total amount $\geq 3 \mu \mathrm{g}$, were considered acceptable and used as an input material to construct the sequencing library. Illumina paired-end library was prepared using the standard TruSeq RNA Sample Prep Kit (Illumina Inc.). Briefly, poly (A) mRNA was enriched from total RNA using Sera-mag Magnetic Oligo (dT) Beads (Thermo Fisher Scientific) according to Illumina manufacturer's instructions. Double-stranded cDNA was generated using the Superscript Double-Stranded cDNA Synthesis Kit (Invitrogen). After that, pair-end RNA-seq libraries were constructed using the Illumina Paired-End Sample Prep kit and were then sequenced on an Illumina HiSeqTM 2000 system. High-quality paired-end reads with a length of $200 \mathrm{bp}$ were obtained by deleting low-quality reads with vague nucleotides and filtering adapters from the raw data.

For transcriptome assembly, a strict Illumina pipeline was used for filtering the raw sequence reads. All reads with adapter sequences, unknown nucleotides comprising more than $10 \%$, and low-quality reads ( $>50 \%$ base with a quality value $Q \leq 5$ in a read) were removed. De novo transcriptome assembly was accomplished from all of these clean reads with the Trinity program. Only sequences with no more than two nucleotide mismatches were used for annotation.

To annotate the transcriptome, assembled sequences were further used as query sequences to BLAST with NCBI nonredundant protein sequence $(\mathrm{Nr})$, SwissProt, Kyoto Encyclopedia of Genes and Genomes (KEGG), Eukaryotic Ortholog Groups (KOG), Pfam, Gene Ontology (GO), and NCBI nucleotide sequences (NT) databases, respectively. The best hit of alignment was used to infer the biological function of assembled transcripts. Additionally, GO terms of assembled transcripts were extracted from the best hits against the $\mathrm{Nr}$ and Pfam using the Blast2GO. After acquiring the GO annotation for each assembled transcript, GO functional classification was achieved using WEGO software for all of the transcripts. KEGG pathway annotations were retrieved from the KEGG (http:// www.genome.jp/kegg/) database.

Analysis of the Differentially Expressed Genes of Octoploid Trititrigia Before and After Aphid Infestation. Using the de novo assembled transcriptome data as a reference sequence, the clean reads of each sample were mapped to this reference sequence using the Bowtie 2, allowing no more than two nucleotide mismatches. The gene expression levels were determined by the numbers of reads uniquely mapped to the specific gene and the total number of uniquely mapped reads in the sample and calculated using the FPKM method (fragments per $\mathrm{kb}$ per million). Using edgeR software, differentially expressed genes (DEGs) were determined between the damaged and intact octoploid Trititrigia head libraries, respectively, and the DEGs were defined as significant based on a false discovery rate (FDR) of $\leq 0.05$ and an absolute value of $\log 2$ ratio $\geq 1$.

The terpene synthase (TPS) genes that were prominently and differentially expressed in the expression profile data were chosen for validation by reverse transcription-quantitative PCR (RT-qPCR) and gene-specific primers were designed by Primer Premier 5. All primer pairs for these qPCR were deposited in additional (Table S1). The qPCR used a Top Green qPCR SuperMix (TransScript) in $20 \mu \mathrm{L}$ reactions according to the manufacturer's instructions. qPCR was performed using the LightCycler96 Real-time PCR System. The qPCR amplification was performed in thermocycler conditions starting with $30 \mathrm{~s}$ at $94{ }^{\circ} \mathrm{C}, 5 \mathrm{~s}$ at $94{ }^{\circ} \mathrm{C}, 40$ cycles of $30 \mathrm{~s}$ at 60 ${ }^{\circ} \mathrm{C}$, and $10 \mathrm{~s}$ at $95{ }^{\circ} \mathrm{C}$, followed by $1 \mathrm{~min}$ at $60{ }^{\circ} \mathrm{C}$. The T. aestivum gene TaActin was taken as an internal reference. To calculate the expression abundances of target genes, the method $2^{-\Delta \Delta \mathrm{Ct}}$ for statistics was applied with three biological replicates.

Cloning and Analysis of the S-Linalool Synthase Gene. The octoploid Trititrigia S-linalool synthase gene (OtLIS) sequence was amplified by RT-PCR using the octoploid Trititrigia total RNA as a template. The cDNA was synthesized by a TaKaRa PrimeScript RTPCR Kit (Dalian, China) and a negative control was set up. The primers were designed according to the transcriptome sequence (Table S1). The full-length ORF of OtLIS was cloned by PCR whose amplification was performed using TaKaRa Tks Gflex DNA Polymerase (Dalian, China) in $50 \mu \mathrm{L}$ reactions according to the manufacturer's instructions. The nested PCR amplification was performed in conditions starting with $60 \mathrm{~s}$ at $94{ }^{\circ} \mathrm{C}, 30$ cycles of $10 \mathrm{~s}$ at $98{ }^{\circ} \mathrm{C}, 15 \mathrm{~s}$ at $55^{\circ} \mathrm{C}$, and $60 \mathrm{~s}$ at $68^{\circ} \mathrm{C}$. The isoelectric point (pI) and molecular weight $(\mathrm{Mw})$ of OtLIS were analyzed by the ExPASy website (http://web.expasy.org/compute_pi/). The proteincoding region of the OtLIS gene was searched by ORF Finder (http://www.ncbi.nlm.nih.gov/projects/gorf/). Multiple sequence alignment of TPS protein sequences was performed by CLUSTALW. To reconstruct the phylogenetic relationship, the neighbor-joining (NJ) tree was conducted using MEGA7.0 and 1000 replicates of bootstrap analyses.

Functional Expression and Enzyme Activity Analysis of OtLIS. The ORF of OtLIS was connected to the prokaryotic expression vector pet32a $(+)$, and the plasmid was transformed into DH5a cells. The final plasmid was transformed into an Escherichia coli dialytic cell-free protein expression system (TSINGKE), inoculated at $25{ }^{\circ} \mathrm{C}$ for $16 \mathrm{~h}$, and the protein was expressed according to the instructions. The supernatant was checked by sodium dodecyl sulfate polyacrylamide gel electrophoresis (SDS-PAGE). Enzyme activity analysis was performed according to the procedure described by Huang et al. ${ }^{29}$ GPP, FPP, and GGPP were used as substrates for enzyme assays. The enzyme activity products were analyzed by GCMS.

\section{RESULTS}

Resistance Assessment, Volatile Collection, and Identification of Octoploid Trititrigia. According to the 2 year field observation on aphid population growth over time, significant decreases of alate and apterous $S$. avenae abundances were observed in octoploid Trititrigia plants compared to the triticale and the control (Figure 1), which 
(A)

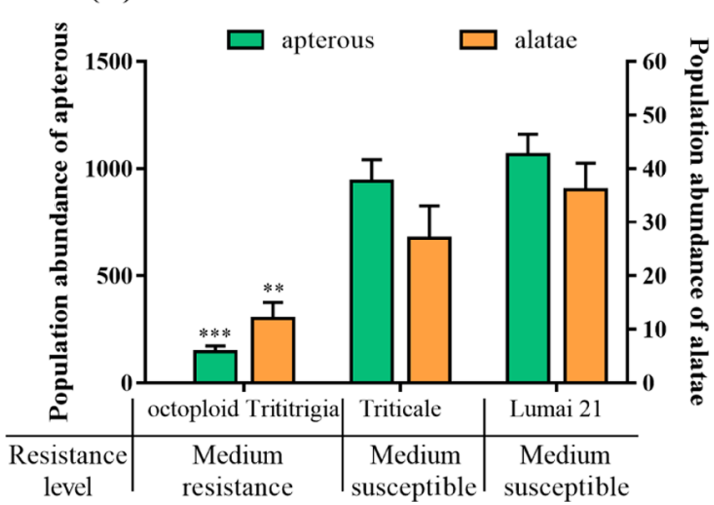

(B)

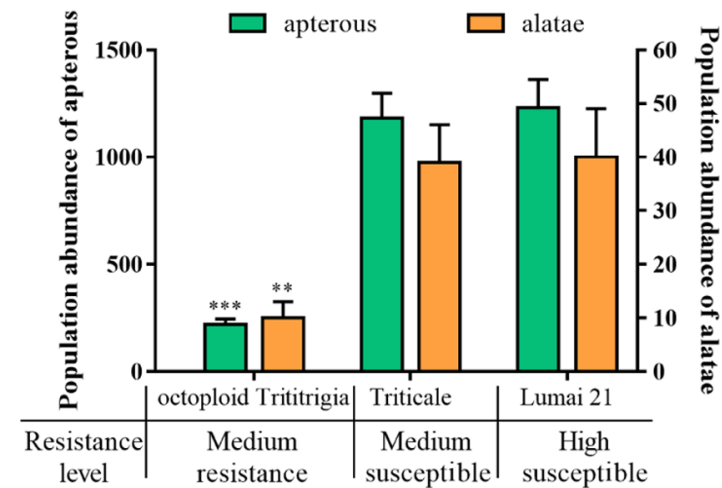

Figure 1. Field tests of the octoploid Trititrigia resistance level to Sitobion avenae both in 2016 (A) and in 2017 (B). The abundances of apterous aphids and alatae were analyzed separately. The left $Y$-axis represents the abundance of the apterous and the right $Y$-axis represents the abundance of alatae. Values are mean \pm standard error. $* *, P<0.01$ and $* * *, P<0.001$.

revealed that $S$. avenae might have nonpreference performance with medium resistance to octoploid Trititrigia during the wheat filling stage (Figure 1). GC-MS analysis showed that the release of seven volatile compounds changed after $S$. avenae infested octoploid Trititrigia, including $S$-linalool, $\delta$-cadinene, (E)-nerolidol, ent-kaurene, $\beta$-Myrcene, $(E)$-3,8-dimethyl-1,4,7nonatriene (DMNT), and $\beta$-caryophyllene. Among them, the amount of S-linalool increased significantly after aphid infestation (Figure 2). So, we focused on linalool and analyzed its resistance properties thereafter.

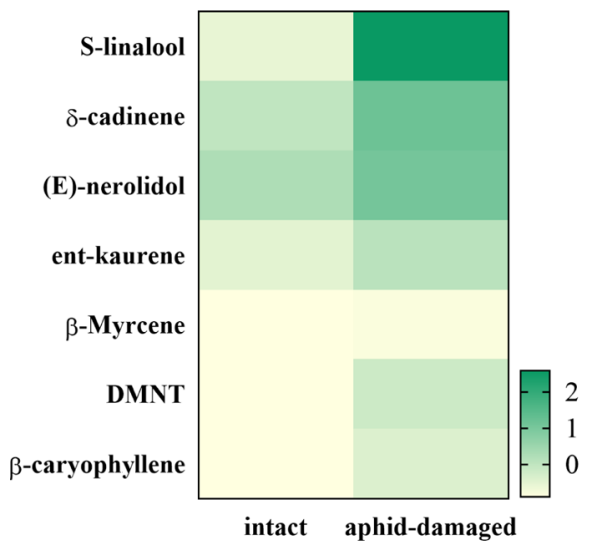

Figure 2. Peak areas of volatile organic compounds (VOCs) emitted from intact and aphid-damaged wheat heads. The value was standardized by the $Z$-score.

Response of S. avenae to S-Linalool. The EAG response of $S$. avenae to $S$-linalool had significant differences among the five concentrations $(F=4.742, P=0.006)$, and the highest EAG response was obtained when the concentration was 1 $\mu \mathrm{g} \cdot \mu \mathrm{L}^{-1}$ (Figure 3A). Olfactory response tests showed that 1 $\mu \mathrm{g} \cdot \mu \mathrm{L}^{-1}(W=-3.385, P<0.05)$ and $10 \mu \mathrm{g} \cdot \mu \mathrm{L}^{-1}(W=$ $-3.352, P<0.01)$ of $S$-linalool had a significant repellent effect on $S$. avenae (Figure 3B).

Strategy for De Novo Assembly and Transcriptomic Data Analysis. We used the samples of the octoploid Trititrigia head and produced $42.6 \mathrm{~Gb}$ of clean data. We obtained 243718 unigenes after sequence assembly. The total length of the transcribed sequence was $203088986 \mathrm{bp}$, the average length was $833 \mathrm{bp}$, and the N50 size was $1041 \mathrm{bp}$ with
47.09\% GC content. The assembled transcript sequence lengths ranged from 300 to $3000 \mathrm{bp}$, and the dominant length of the sequences was $300-400 \mathrm{bp}$ (Figure S1). In addition, the number of transcripts of sequence length $\geq 3000$ bp was 3863 .

For a comprehensive understanding of the role of the interesting gene, the detected genes were functionally annotated in the databases of NR public database, Swissport, GO, KEGG, KOG, and NT. A total of 182348 (74.8\%) unigenes could be annotated into at least one of the six databases. Among them, 85145 (34.94\%), 73806 (40.48\%), 53526 (21.96\%), 125347 (51.47\%), 138277 (56.74\%), and 68267 (28.01\%) unigenes were BLAST to GO, KEGG, KOG, NR, NT, and Swissport, respectively.

Based on the FPKM method, the transcript abundance of each gene from the intact octoploid Trititrigia heads and the aphid-damaged heads was analyzed. A total of 233023 DEGs were identified between the intact and damaged octoploid Trititrigia head libraries. These DEGs were assigned to GO (gene ontology) classes to explore their functions (Figure S2). Out of these DEGs, significantly upregulated genes in octoploid Trititrigia heads induced by $S$. avenae were identified. Candidate resistance genes were identified from overlapping TPS genes; TPS functions as a central regulator in resistance against $S$. avenae (Figure 4).

Candidate Genes Related to S-Linalool Biosynthesis. The levels of transcripts encoding the S-linalool synthase (LIS) of aphid-damaged octoploid Trititrigia heads were significantly higher than those of intact octoploid Trititrigia heads. These were further validated using RT-qPCR. The levels of genes encoding LIS were significantly higher in the aphid-damaged octoploid Trititrigia than those of intact octoploid Trititrigia heads (Figure 5). Based on RT-qPCR results, the expression profiles of the seven genes were identified by the Illumina sequencing or RT-qPCR analysis, but the accurate folding change of the two methods was biased. This suggested relative rationality and accuracy of the transcriptome analysis in the present study. The expression of TR127690_c3_g3 was most significantly increased after aphid infection and was selected for further study.

Characterization and Phylogenetic Analysis of OtLIS. GC-MS analysis showed that S-linalool was the only volatile compound with a significant difference in the amount emitted from the aphid-damaged octoploid Trititrigia heads. We cloned the complete ORF of OtLIS by nested PCR. The 
(A)

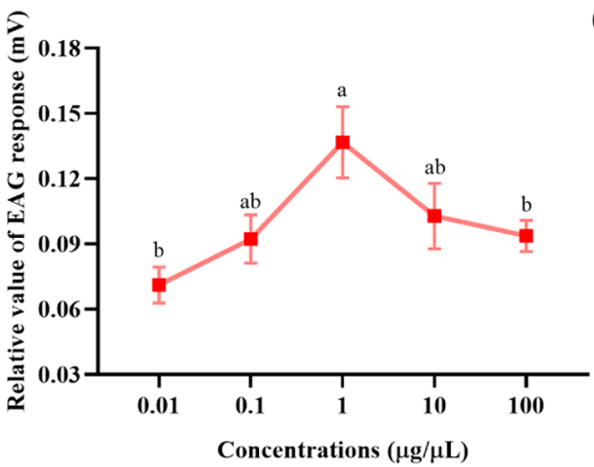

(B)

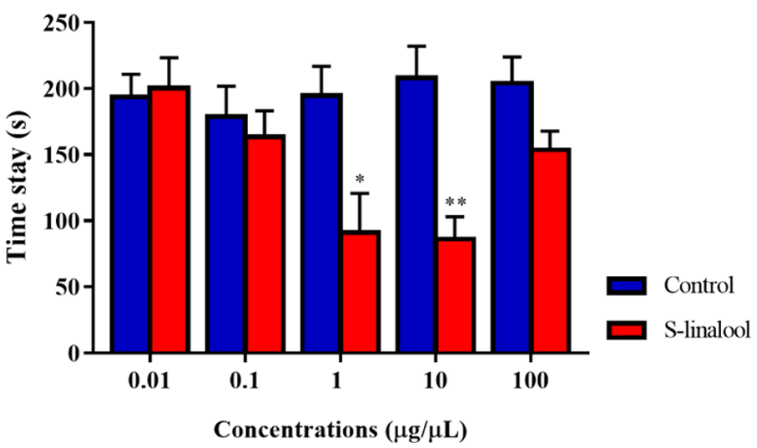

Figure 3. Responses of Sitobion avenae to S-linalool. (A) Electroantennography responses (mean \pm SE) of S. avenae to S-linalool. Error bars represent the standard errors of six replicates. Lowercase letters indicate the significant difference $(P<0.05)$. (B) Olfactory responses of the $S$. avenae to S-linalool. * and ** indicate the significant difference $(P<0.05)$ and the extremely significant difference $(P<0.01)$ between treatment and control.

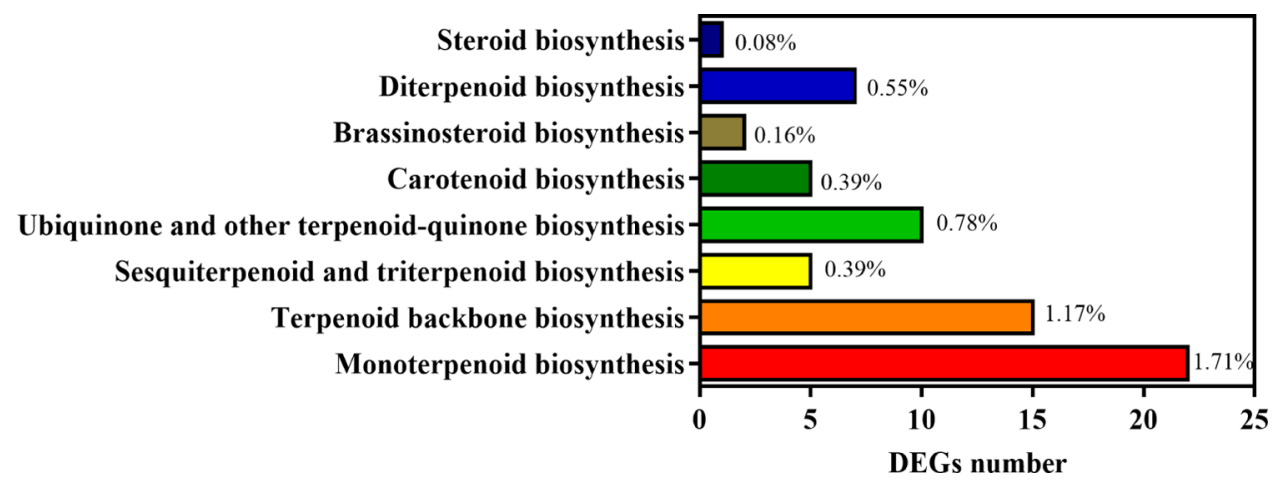

Figure 4. Analysis of differentially expressed genes involved in terpenoid anabolism in the KEGG pathway between intact octoploid Trititrigia heads and aphid-damaged heads.

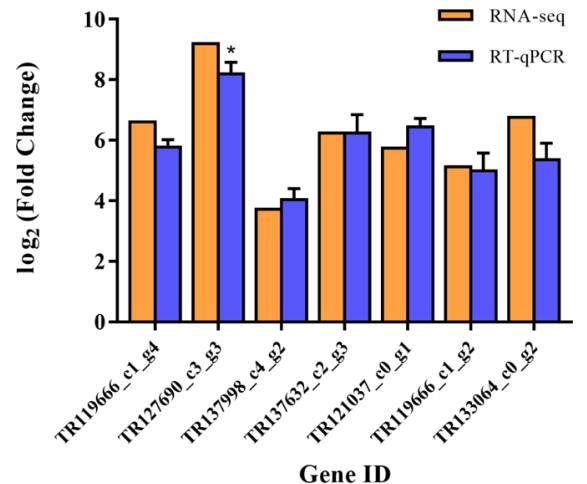

Figure 5. Expression level of S-linalool biosynthesis genes using the RT-qPCR, in comparison to corresponding data detected in the RNASeq. The relative expression ratio of each DEG is presented in a $\log 2$ value of the aphid-damaged head vs the intact octoploid Trititrigia head libraries. The values for mean $\pm \mathrm{SE}(n=3)$. Data were normalized to the TaActin gene and vertical bars indicate standard deviation. * indicates the significant difference $(P<0.05)$ between the relative expression of genes.

complete ORF of OtLIS was $1680 \mathrm{bp}$, within a predicted Mw of $64.19 \mathrm{kDa}$, coding 559 amino acids (aa), and had a pI of 5.9. The amino acid sequence alignment results show that OtLIS contained an aspartate-rich region DDxxD motif (Figure 6). The sequence data have been submitted to the GenBank database under accession number MZ388428.

OtLIS clustered with other angiosperm monoterpene synthases (TPS-g family) based on the phylogenetic relation- ship analysis (Figure 7). There was no difference in the phylogenetic relationship of the OtLIS family from that of the current plant. Furthermore, the LIS of the close relatives almost clustered into a group, indicating that they had a close evolutionary relationship in related plant species other than distant plant species.

Functional Validation of OtLIS. The function of OtLIS was analyzed according to the potential substrate (GPP, FPP, GGPP); GC-MS results showed that recombinant protein OtLIS could catalyze the formation of S-linalool from GPP and $(E)$-nerolidol from FPP in vitro (Figure 8). No enzyme activity was detected in the reaction system with GGPP substrates.

\section{DISCUSSION}

Breeding insect pest-resistant varieties is an efficient pestcontrolling strategy for agricultural crops. Screening insect pest-resistant germplasm and mining-resistant genes are the basis of breeding-resistant varieties. Wheat is the major crop worldwide that is severely damaged by aphids, which leads to yield and quality reduction, while the conventional varieties of wheat lack aphid-resistant genes. The medium resistance of octoploid Trititrigia to $S$. avenae was determined since the numbers of $S$. avenae including the apterous aphids and alatae on the octoploid Trititrigia plant were far less than those of others, and the resistance mechanism was likely nonpreferable (Figure 1). It indicated that the octoploid Trititrigia plant may produce volatile chemical compounds that had a repellent effect on S. avenae in the wheat canopy. Our study showed that S-linalool was emitted from both intact octoploid Trititrigia 

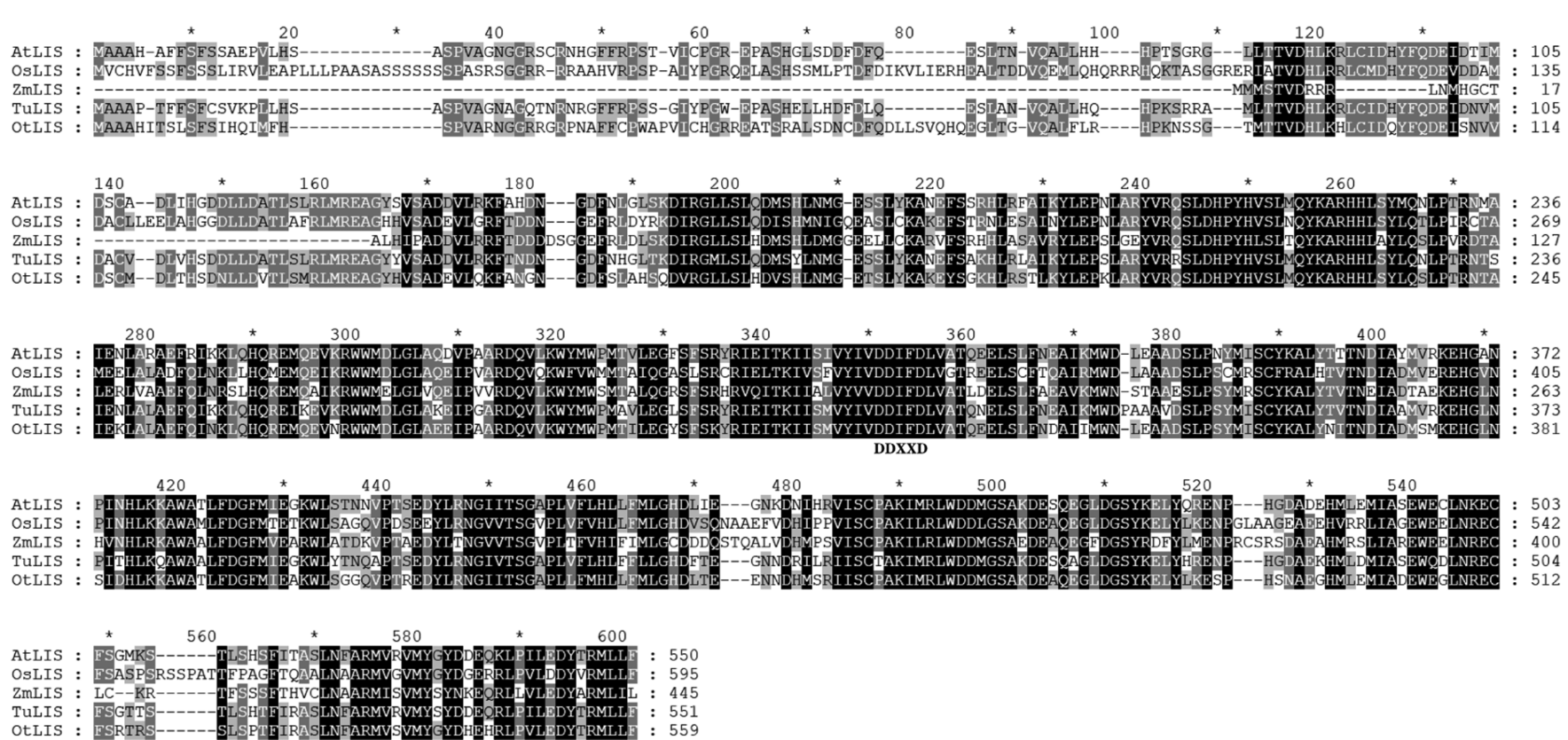

Figure 6. Alignment of OtLIS, Oryza sativa LIS (XP_015623808), Zea mays LIS (AQK60700), Triticum Urartu NES1 (EMS50987), and Arabidopsis thaliana LIS (NP_176361) amino acid sequences. The highly conserved regions are DDxxD. The alignment was performed by CLUSTALW.

Tree scale: 0.1

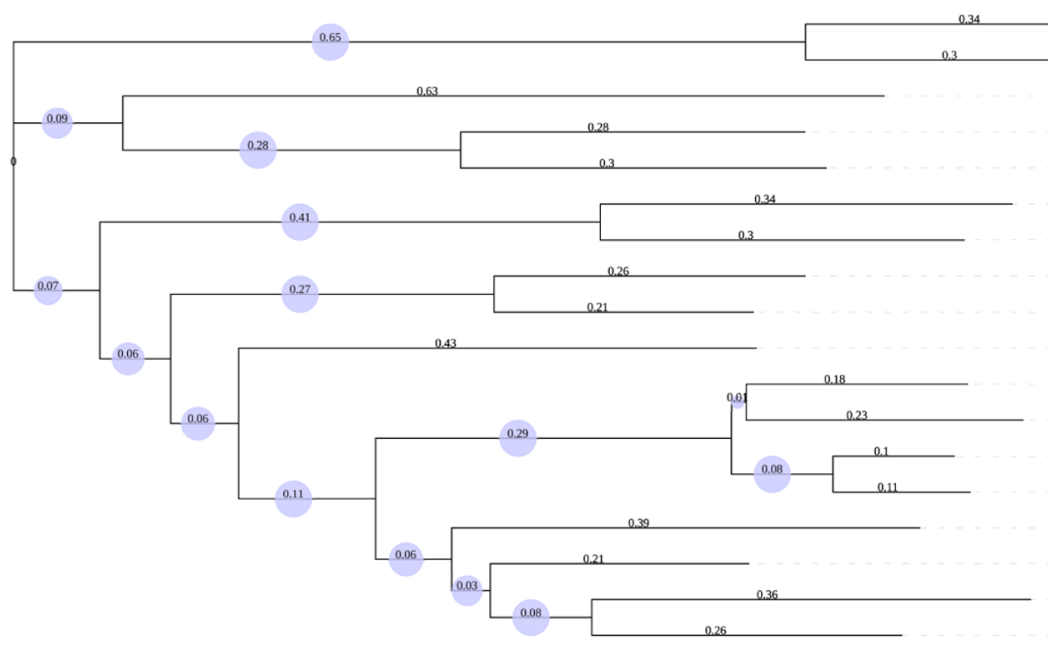

Solanum lycopersicum CPS
Cucurbita maxima CPS1
Arabidopsis thaliana TPS04
Arabidopsis thaliana ent-KS
Stevia rebaudiana KS
Arabidopsis thaliana TPS07
Arabidopsis thaliana TPS01
Arabidopsis thaliana TPS03
Arabidopsis thaliana TPS02
Phaseolus lunatus TPS3
Oryza sativa LIS
Zea mays LIS
Triticum urartu NES
Octoploid Trititrigia LIS
Arabidopsis thaliana TPS14
Vitis vinifera LIN/NER/GERS
Phaseolus lunatus TPS4
Medicago truncatula TPS3

Colored ranges

TPS-g

TPS-b

TPS-a

TPS-e/f

TPS-c

Figure 7. Phylogenetic tree of OtLIS together with known TPSs from other plants. OtLIS fell in the cluster of angiosperm monoterpene synthases (TPS-g). The functional descriptions and accession numbers of these genes are listed in Table S2.

heads and $S$. avenae-damaged heads from which a higher amount was emitted. It revealed that the $S$. avenae feeding could induce the biosynthesis of the volatile terpenoid to produce a large amount of S-linalool, which enhanced the defensive effect on $S$. avenae.

Previous studies have shown that S-linalool was the most abundant volatile emitted from the pest-damaged rice and cotton plants and showed direct or indirect defense against herbivorous insect pests. ${ }^{29,42}$ Genetical engineering Arabidopsis thaliana plants released S-linalool that repelled M. persicae. ${ }^{48}$ Additionally, the field studies conducted with OsLIS-silenced rice plants showed that inducible (3S)-linalool attracted the predators, parasitoids, as well as chewing herbivores but repelled the brown planthopper $N$. lugens. ${ }^{41}$ In this study, we demonstrated that S-linalool (1 and $10 \mu \mathrm{g} \cdot \mu \mathrm{L}^{-1}$ ) had a remarkable repellent effect on $S$. avenae by the four-arm olfactory test. This could provide a new semiochemical for exogenous application with aphid avoidance in the field.

Some TPSs have been identified, and even successfully used to increase direct plant defense or $^{29,49}$ or indirect plant defense by attracting natural enemies of the herbivores. ${ }^{50-52} \mathrm{~A}$ few TPSs have been discovered in the field crops, ${ }^{13,53,54}$ which have greatly promoted the understanding of the mediation of terpene compound biosynthesis. Meanwhile, studies have reported that the expression levels of TPSs were significantly correlated with pest-defense mechanisms in the plants. ${ }^{29,55,56}$ Then, we used transcriptomic data of the intact and aphiddamaged octoploid Trititrigia heads to screen the regulatory genes in the terpene biosynthetic pathway. ${ }^{47}$ This assembled transcriptome could be used as a database of the wheat 


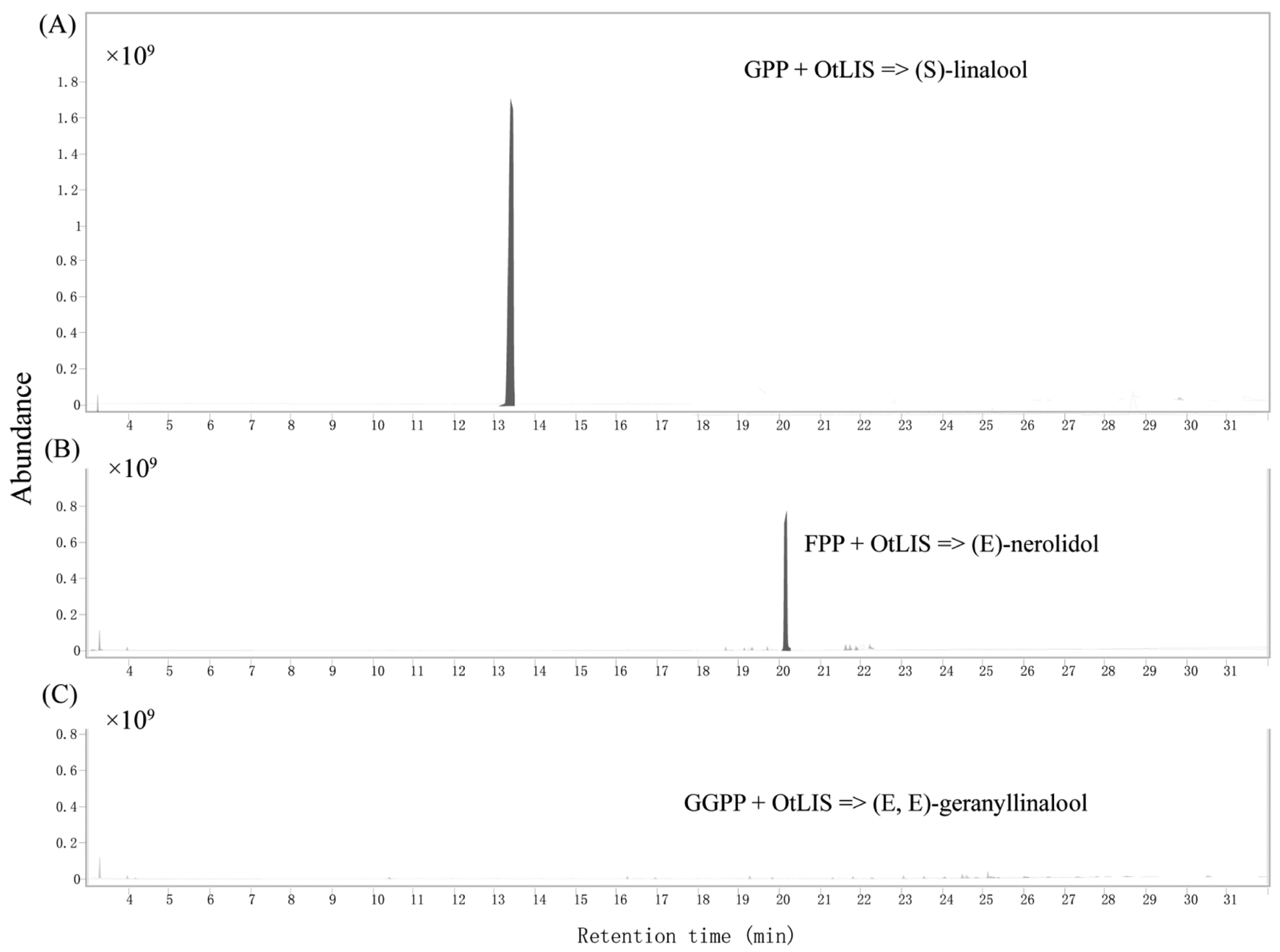

Figure 8. GC-MS analysis of the volatile products of recombinant OtLIS. The protein was heterologously expressed in E. coli. The expression products of OtLIS after the incubation with GPP (A), FPP (B), and GGPP (C).

germplasm to search for high-quality aphid-resistant genes and provide more molecular resources for future functional characterization analysis of aphid resistance.

The emission of the volatile terpenoid S-linalool was higher in aphid-fed octoploid Trititrigia heads than untreated heads. At the same time, the transcripts of S-linalool synthase genes were also the most abundant. However, little has been known about the molecular mechanisms controlling S-linalool biosynthesis in wheat. In this study, the OtLIS sequence was obtained, and amino acid sequence alignment of OtLIS, Oryza sativa LIS (XP_015623808), Zea mays LIS (AQK60700), Triticum urartu LIS (EMS50987), and A. thaliana LIS (NP 176361) revealed the typical conserved motifs and domains of the specific terpene synthase (Figure 6). OtLIS contained an aspartate-rich region $\mathrm{DDxxD}$ motif, which was described as a function involved in substrate binding to coordinate divalent metal ions. ${ }^{57}$ Phylogenetic analysis demonstrated that the OtLIS and TuNES1 were highly homologous. The results could help to provide a deeper understanding of how changes in the gene expression are related to the volatile terpenoid S-linalool changes in the octoploid Trititrigia heads.

Plants have evolved a series of defense mechanisms against biotic stressors. ${ }^{58}$ In addition to terpenes, plants release a spectrum of VOCs including fatty acids, phenylpropanoids, green leaf volatiles, and glucosinolate breakdown products. $^{59-61}$ At present, several pest management strategies have been developed based on VOC-mediated plant-insect interactions, such as push-pull systems, synthetic odorant traps, and crop cultivars with modified VOC profiles. ${ }^{62}$ Our study is of great significance, showing that OtLIS, which encodes terpene synthase, confers resistance to a wheat aphid by S-linalool emission. The deployment of OtLIS for breeding aphid-resistant wheat cultivars could alleviate both the yield loss and the virus transmission problems for growers.

\section{ASSOCIATED CONTENT}

\section{SI Supporting Information}

The Supporting Information is available free of charge at https://pubs.acs.org/doi/10.1021/acs.jafc.1c05978.

Primers used in the quantitative real-time PCR and nested PCR and the complete ORF of octoploid Trititrigia S-linalool synthase gene, protein sequences used for phylogenetic analysis of the plant TPSs, length distribution of the assembled transcript sequences of octoploid Trititrigia heads, and the number of DEGs associated with biological processes in Gene Ontology (GO) classification (PDF) 


\section{AUTHOR INFORMATION}

\section{Corresponding Author}

Yong Liu - College of Plant Protection, Shandong Agricultural University, Taian, Shandong 271018, China; Phone: Tel: +86538 8248208; Email: liuyong@sdau.edu.cn

\section{Authors \\ Yidi Zhan - College of Plant Protection, Shandong Agricultural University, Taian, Shandong 271018, China; (1) orcid.org/0000-0003-0516-4701 \\ Lei Zhao - College of Plant Protection, Shandong Agricultural University, Taian, Shandong 271018, China \\ Xiaojing Zhao - College of Plant Protection, Shandong Agricultural University, Taian, Shandong 271018, China \\ Jiahui Liu - College of Plant Protection, Shandong Agricultural University, Taian, Shandong 271018, China; Functional and Evolutionary Entomology, Terra, Gembloux Agro-Bio Tech, Liege University, 5030 Gembloux, Belgium \\ Frederic Francis - College of Plant Protection, Shandong Agricultural University, Taian, Shandong 271018, China; Functional and Evolutionary Entomology, Terra, Gembloux Agro-Bio Tech, Liege University, 5030 Gembloux, Belgium}

Complete contact information is available at:

https://pubs.acs.org/10.1021/acs.jafc.1c05978

\section{Author Contributions}

${ }^{\S}$ Y.Z. and L.Z. should be considered joint first author.

\section{Funding}

This research was supported by grants from the Natural Science Foundation of Shandong Province (ZR2020MC121) and the National Key R\&D Program of China (2017YFD0201705).

\section{Notes}

The authors declare no competing financial interest.

\section{ACKNOWLEDGMENTS}

This research was supported by grants from the Natural Science Foundation of Shandong Province (ZR2020MC121) and the National Key R\&D Program of China (2017YFD0201705).

\section{ABBREVIATIONS USED}

DMNT, (E)-3,8-dimethyl-1,4,7-nonatriene; EAG, electroantennography; FPKM, fragments per $\mathrm{kb}$ per million; FPP, farnesyl diphosphate; GGPP, geranylgeranyl diphosphate; GPP, geranyl diphosphate; LIS, S-linalool synthase; MEP, 2c-methyl-d-erythritol 4-phosphate; MVA, mevalonate; Mw, molecular weight; OtLIS, octoploid Trititrigia S-linalool synthase; pI, isoelectric point; PTFE, poly(tetrafluoroethylene); TPS-a, sesquiterpene synthases; TPS-b, cyclic monoterpene and hemiterpene synthases; TPS-c, copalyl diphosphate synthases; TPS-d, gymnosperm-specific terpene synthases; TPS-e, ent-kaurene synthases; TPS-f, other diterpene synthases; TPS-g, acyclic monoterpenes; TPS-h, specific to lycopod Selaginella moellendorffii; TPSs, terpene synthases; VOCs, volatile organic compounds

\section{REFERENCES}

(1) Godfray, H. C. J.; Beddington, J. R.; Crute, I. R.; Haddad, L.; Lawrence, D.; Muir, J. F.; Pretty, J.; Robinson, S.; Thomas, S. M.; Toulmin, C. Food security: the challenge of feeding 9 billion people. Science 2010, 327, 812-818.
(2) Wang, G.; Cui, L. L.; Dong, J.; Francis, F.; Liu, Y.; Tooker, J. F. Combining intercropping with semiochemical releases: optimization of alternative control of Sitobion avenae in wheat crops in China. Entomol. Exp. Appl. 2011, 140, 189-195.

(3) Ma, G.; Rudolf, V. H. W.; Ma, C. S. Extreme temperature events alter demographic rates, relative fitness, and community structure. Global Change Biol. 2015, 21, 1794-1808.

(4) Zhang, K.; Pan, Q.; Yu, D.; Wang, L. M.; Liu, Z. Z.; Li, X.; et al. Systemically modeling the relationship between climate change and wheat aphid abundance. Sci. Total Environ. 2019, 674, 392-400.

(5) Quillec, F. L.-L.; Tanguy, S.; Dedryver, C. A. Aerial flow of barley yellow dwarf viruses and of their vectors in western France. Ann. Appl. Biol. 1995, 126, 75-90.

(6) Fiebig, M.; Poehling, H. M.; Borgemeister, C. Barley yellow dwarf virus, wheat, and Sitobion avenae: a case of trilateral interactions. Entomol. Expe. Appl. 2004, 110, 11-21.

(7) Liu, Y.; Wang, W. L.; Guo, G. X.; Ji, X. L. Volatile emission in wheat and parasitism by Aphidius avenae after exogenous application of salivary enzymes of Sitobion avenae. Entomol. Exp. Appl. 2009, 130, 215-221.

(8) Rozas, L. J. A.; Vieira, F. G. Genome sequence of the pea aphid Acyrthosiphon pisum. PLoS Biol. 2010, 8, e1000313.

(9) Wang, K.; Liu, J. H.; Zhan, Y. D.; Liu, Y. A new slow-release formulation of methyl salicylate optimizes the alternative control of Sitobion avenae (Fabricius) (Hemiptera: Aphididae) in wheat fields. Pest Manag. Sci. 2019, 75, 676-682.

(10) Smith, C. M. Plant Resistance Against Pests: Issues and Strategies. In Integrated Pest Management: Potential, Constraints and Challenges, Koul, O.; Dhaliwal, G. S.; Cuperus, G., Eds.; CABI: Oxfordshire, UK, 2004; pp 147-167.

(11) Tagu, D.; Klingler, J. P.; Moya, A.; Simon, J. C. Early progress in aphid genomics and consequences for plant-aphid interactions studies. Mol. Plant-Microbe Interact. 2008, 21, 701.

(12) Elek, H.; Werner, P.; Smart, L. E.; Gordon-Weeks, R.; Nadasy, M.; Pickett, J. A. Aphid resistance in wheat varieties. Commun. Agric. Appl. Biol. Sci. 2009, 74, 233-241.

(13) Bruce, T. J. A.; Aradottir, G. I.; Smart, L. E.; Martin, J. L.; Caulfield, J. C.; Doherty, A.; Sparks, C. A.; Woodcock, C. M.; Birkett, M. A.; Napier, J. A.; Jones, H. D.; Pickett, J. A. The first crop plant genetically engineered to release an insect pheromone for defence. Sci. Rep. 2015, 5, No. 11183.

(14) Zhang, Y.; Li, Z. X.; Yu, X. D.; Fan, J.; Pickett, J. A.; Jones, H. D.; Zhou, J. J.; Birkett, M. A.; Caulfield, J.; Napier, J. A.; Zhao, G. Y.; Cheng, X. G.; Shi, Y.; Bruce, T. J. A.; Xia, L. Q. Molecular characterization of two isoforms of a farnesyl pyrophosphate synthase gene in wheat and their roles in sesquiterpene synthesis and inducible defence against aphid infestation. New Phytol. 2015, 206, 1101-1115. (15) Xia, L. Q.; Ma, Y. Z.; He, Y.; Jones, H. D. GM wheat development in China: current status and challenges to commercialization. J. Exp. Bot. 2012, 63, 1785-1790.

(16) Yu, X. D.; Wang, G. P.; Huang, S. L.; Ma, Y. Z.; Xia, L. Q. Engineering plants for aphid-resistance: current status and future perspectives. Theor. Appl. Genet. 2014, 127, 2065-2083.

(17) Liu, F.; Kang, Z.; Tan, X.; Fan, Y.; Tian, H.; Liu, T. Physiology and defense responses of wheat to the infestation of different cereal aphids. J. Integr. Agr. 2020, 19, 1464-1474.

(18) Aradottir, G. I.; Crespo-Herrera, L. Host plant resistance in wheat to barley yellow dwarf viruses and their aphid vectors: a review. Curr. Opin. in Insect Sci. 2021, 45, 59-68.

(19) Pickett, J. A.; Aradottir, G. I.; Birkett, M. A.; Bruce, T. J. A.; Chamberlain, K.; Khan, Z. R.; Midega, C. A. O.; Smart, L. E.; Woodcock, C. M. Aspects of insect chemical ecology: exploitation of reception and detection as tools for deception of pests and beneficial insects. Physiol. Entomol. 2012, 37, 2-9.

(20) Yu, X. D.; Pickett, J.; Ma, Y. Z.; Bruce, T.; Napier, J.; Jones, H. D.; Xia, L. Q. Metabolic engineering of plant-derived (E)- $\beta$-farnesene synthase genes for a novel type of aphid-resistant genetically modified crop plant. J. Integr. Plant Biol. 2012, 54, 282-299. 
(21) Liu, Y.; Liu, J.; Zhou, H.; Chen, J. Enhancement of Natural Control Function for Aphids by Intercropping and Infochemical Releasers in Wheat Ecosystem. In Integrative Biological Control: Progress in Biological Control, Gao, Y.; Hokkanen, H.; MenzlerHokkanen, I., Eds.; Springer: Cham, 2020; pp 85-116.

(22) Goggin, F. L. Plant aphid interactions: molecular and ecological perspectives. Curr. Opin. Plant Biol. 2007, 10, 399-408.

(23) Smith, C. M.; Boyko, E. V. The molecular bases of plant resistance and defence responses to aphid feeding: current status. Entomol. Exp. Appl. 2007, 122, 1-16.

(24) Smith, C. M.; Chuang, W. P. Plant resistance to aphid feeding: behavioral, physiological, genetic and molecular cues regulate aphid host selection and feeding. Pest Manag. Sci. 2014, 70, 528-540.

(25) Liu, J.; Zhao, X.; Zhan, Y.; Wang, K.; Francis, F.; Liu, Y. New slow release mixture of (E)- $\beta$-farnesene with methyl salicylate to enhance aphid biocontrol efficacy in wheat ecosystem. Pest Manag. Sci. 2021, 77, 3341-3348.

(26) Francis, F.; Lognay, G.; Haubruge, E. Olfactory responses to aphid and host plant volatile releases: $\mathrm{E}-\beta$-farnesene an effective kairomone for the predator Adalia bipunctata. J. Chem. Ecol. 2004, 30, $741-755$.

(27) Bruce, T. J.; Birkett, M. A.; Blande, J.; Hooper, A. M.; Martin, J. L.; Khambay, B.; Prosser, I.; Smart, L. E.; Wadhams, L. J. Response of economically important aphids to components of Hemizygia petiolata essential oil. Pest Manag. Sci. 2005, 61, 1115-1121.

(28) Halbert, S. E.; Corsini, D.; Wiebe, M.; Vaughn, S. F. Plantderived compounds and extracts with potential as aphid repellents. Ann. Appl. Biol. 2009, 154, 303-307.

(29) Huang, X.-Z.; Xiao, Y. T.; Köllner, T. G.; Jing, W. X.; Kou, J. F.; Chen, J. Y.; Liu, D. F.; Gu, S. H.; Wu, J. X.; Zhang, Y. G.; Guo, Y. Y. The terpene synthase gene family in Gossypium hirsutum harbors a linalool synthase GhTPS12 implicated in direct defence responses against herbivores. Plant, Cell Environ. 2017, 41, 261-274.

(30) Lichtenthaler, H. K. The 1-Deoxy-D-Xylulose-5-Phosphate pathway of isoprenoid biosynthesis in plants. Annu. Rev. Plant Physiol. Plant Mol. Biol. 1999, 50, 47-65.

(31) Carretero-Paulet, L.; Ahumada, I.; Cunillera, N.; RodríguezConcepción, M.; Ferrer, A.; Boronat, A.; Campos, N. Expression and molecular analysis of the Arabidopsis DXR gene encoding 1-deoxy-Dxylulose 5-phosphate reductoisomerase, the first committed enzyme of the 2-C-methyl-D-erythritol 4-phosphate pathway. Plant Physiol. 2002, 129, 1581-1591.

(32) Rodríguez-Concepción, M.; Borona, A. Elucidation of the methylerythritol phosphate pathway for isoprenoid biosynthesis in bacteria and plastids. A metabolic milestone achieved through genomics. Plant Physiol. 2002, 130, 1079-1089.

(33) McGarvey, D. J.; Croteau, R. Terpenoid metabolism. Plant Cell 1995, 7, 1015-1026.

(34) Aubourg, S.; Lecharny, A.; Bohlmann, J. Genomic analysis of the terpenoid synthase (AtTPS) gene family of Arabidopsis thaliana. Mol. Gen. Genomics 2002, 267, 730-745.

(35) Chen, F.; Tholl, D.; Bohlmann, J.; Pichersky, E. The family of terpene synthases in plants: a mid-size family of genes for specialized metabolism that is highly diversified throughout the kingdom. Plant J. 2011, 66, 212-229.

(36) Knudsen, J. T.; Tollsten, L.; Bergström, G. Floral scents-a checklist of volatile compound by head-space techniques. Phytochemistry 1993, 33, 253-280.

(37) Lin, Y. L.; Lee, Y. R.; Huang, W. K.; Chang, S. T.; Chu, F. H. Characterization of $\mathrm{S}-(+)$-linalool synthase from several provenances of Cinnamomum osmophloeum. Tree Genetics Genomes 2014, 10, 7586.

(38) Shimada, T.; Endo, T.; Fujii, H.; Rodríguez, A.; Peña, L.; Omura, M. Characterization of three linalool synthase genes from Citrus unshiu Marc. and analysis of linalool-mediated resistance against Xanthomonas citri subsp. citri and Penicilium italicum in citrus leaves and fruits. Plant Sci. 2014, 229, 154-166.

(39) Zhu, B.-Q.; Cai, J.; Wang, Z. Q.; Xu, X. Q.; Pan, Q. H. Identification of a plastid-localized bifunctional nerolidol/linalool synthase in relation to linalool biosynthesis in young grape berries. Int J. Mol. Sci. 2014, 15, 21992-22010.

(40) Zhou, Y.; Deng, R. F.; Xu, X. L.; Yang, Z. Y. Enzyme catalytic efficiencies and relative gene expression levels of ( $R$ )-linalool synthase and (S)-linalool synthase determine the proportion of linalool enantiomers in Camellia sinensis var. sinensis. J. Agric. Food Chem. 2020, 68, 10109-10117.

(41) Xiao, Y.; Wang, Q.; Erb, M.; Turlings, T. C. J.; Ge, L.; Hu, L.; Li, J.; Han, X.; Zhang, T.; Lu, J.; Zhang, G.; Lou, Y. Specific herbivore-induced volatiles defend plants and determine insect community composition in the field. Ecol. Lett. 2012, 15, 1130-1139.

(42) Yuan, J. S.; Köllner, T. G.; Wiggins, G.; Grant, J.; Zhao, N.; Zhuang, X. F.; Degenhardt, J.; Chen, F. Elucidation of the genomic basis of indirect plant defense against insects. Plant Signal. Behav. 2008, 3, 720-721.

(43) Cui, Y.; Xing, P.; Qi, X.; Bao, Y.; Wang, H.; Wang, R. R.; Li, X. Characterization of chromosome constitution in three wheat Thinopyrum intermedium amphiploids revealed frequent rearrangement of alien and wheat chromosomes. BMC Plant Biol. 2021, 21, No. 129.

(44) Gupta, P. K. Use of Alien Genetic Variation for Wheat Improvement. In Molecular Breeding for Sustainable Crop Improvement; Sustainable Development and BiodiversitySpringer, 2016; Vol. 11.

(45) Du, Y. Z.; Grodowitz, M. J.; Chen, J. Electrophysiological responses of eighteen species of insects to fire ant alarm pheromone. Insects 2019, 10, No. 403.

(46) Zhao, M. Z.; Liu, B.; Sun, Y. Y.; Wang, Y. Y.; Dai, L. L.; Chen, $\mathrm{H}$. Presence and roles of myrtenol, myrtanol and myrtenal in Dendroctonus armandi (Coleoptera: Curculionidae: Scolytinae) and Pinus armandi (Pinales: Pinaceae: Pinoideae). Pest Manag. Sci. 2020, 76, 188-197.

(47) Zhao, L.; Liu, Z.; Zhan, Y.; Liu, Y. De novo assembly and comparative transcriptome analysis revealed the genes that were potentially involved in defensive terpenoids emission in the wheat against the wheat aphid Sitobion avenae (Fabricius). Preprint available at Research Square 2019. DOI: 10.21203/rs.2.15301/v2.

(48) Aharoni, A.; Giri, A. P.; Deuerlein, S.; Griepink, F.; de Kogel, W. J.; Verstappen, F. W. A.; Verhoeven, H. A.; Jongsma, M. A.; Schwab, W.; Bouwmeester, H. J. Terpenoid metabolism in wild-type and transgenic Arabidopsis Plants. Plant Cell 2003, 15, 2866-2884.

(49) Hegde, M.; Oliveira, J. N.; Costa, J. G. D.; Loza-Reyes, E.; Bleicher, E.; Santana, A. E. G.; Caulfield, J. C.; Mayon, P.; Dewhirst, S. Y.; Bruce, T. J. A.; Pickett, J. A.; Birkett, M. A. Aphid antixenosis in cotton is activated by the natural plant defence elicitor cis-jasmone. Phytochemistry 2012, 78, 81-88.

(50) Rasmann, S.; Köllner, T. G.; Degenhardt, J.; Hiltpold, I.; Toepfer, S.; Kuhlmann, U.; Gershenzon, J.; Turlings, T. C. J. Recruitment of entomopathogenic nematodes by insect-damaged maize roots. Nature 2005, 434, 732-737.

(51) Williams, L.; Rodriguez-Saona, C.; Paré, P. W.; CraftsBrandner, S. J. The piercing-sucking herbivores Lygus hesperus and Nezara viridula induce volatile emissions in plants. Arch. Insect. Biochem. 2005, 58, 84-96.

(52) Fontana, A.; Held, M.; Fantaye, C. A.; Turlings, T. C.; Degenhardt, J.; Gershenzon, J. Attractiveness of constitutive and herbivore-induced sesquiterpene blends of maize to the parasitic wasp Cotesia marginiventris (Cresson). J. Chem. Ecol. 2011, 37, 582-591.

(53) Richter, A.; Seidl-Adams, I.; Köllner, T.; Schaff, C.; Tumlinson, J. H.; Degenhardt, J. A Small, differentially regulated family of farnesyl diphosphate synthases in maize (Zea mays) provides farnesyl diphosphate for the biosynthesis of herbivore-induced sesquiterpenes. Planta 2015, 241, 1351-1361.

(54) Liu, D.; Huang, X.; Jing, W.; An, X.; Zhang, Q.; Zhang, H.; Zhou, J. J.; Zhang, Y. J.; Guo, Y. Y. Identification and functional analysis of two P450 enzymes of Gossypium hirsutum involved in DMNT and TMTT biosynthesis. Plant Biotechnol. J. 2018, 16, 581590.

(55) Richter, A.; Schaff, C.; Zhang, Z.; Lipka, A. E.; Tian, F.; Köllner, T. G.; Schnee, C.; Preiß, S.; Irmisch, S.; Jander, G.; Boland, 
W.; Gershenzon, J.; Buckler, E. S.; Degenhardt, J. Characterization of biosynthetic pathways for the production of the volatile homoterpenes DMNT and TMTT in Zea mays. Plant Cell 2016, 28, 2651-2665.

(56) Li, F.; Li, W.; Lin, Y. J.; Pickett, J. A.; Birkett, M. A.; Wu, K. M.; Wang, G. R.; Zhou, J. J. Expression of lima bean terpene synthases in rice enhances recruitment of a beneficial enemy of a major rice pest. Plant, Cell Environ. 2017, 41, 111-120.

(57) Degenhardt, J.; Hiltpold, I.; Tobias, G. K.; Frey, M.; Gierl, A.; Gershenzon, J.; Hibbard, B. E.; Ellersieck, M. R.; Turlings, T. C. J. Restoring a maize root signal that attracts insect-killing nematodes to control a major pest. Proc. Natl. Acad. Sci. U.S.A. 2009, 106, 1321313218.

(58) Kessler, A.; Baldwin, I. T. Plants responses to insect herbivory: the emerging molecular analysis. Annu. Rev. Plant Biol. 2002, 53, 299328.

(59) Dudareva, N.; Pichersky, E. Metabolic engineering of plant volatiles. Curr. Opin. Biotechnol. 2008, 19, 181-189.

(60) Skoczek, A.; Piesik, D.; Wenda-Piesik, A.; Buszewski, B.; Bocianowski, J.; Wawrzyniak, M. Volatile organic compounds released by maize following herbivory or insect extract application and communication between plants. J. Appl. Entomol. 2017, 141, 630643.

(61) Zhang, Z.; Liu, Y.; Portaluri, V.; Woodcock, C.; Pickett, J. A.; Wang, S.; Zhou, J. J. Chemical Identity and Functional Characterization of Semiochemicals That Promote the Interactions between Rice Plant and Rice Major Pest Nilaparvata lugens. J. Agric. Food Chem. 2021, 69, 4635-4644.

(62) Zhou, S.; Jander, G. Molecular ecology of plant volatiles in interactions with insect herbivores. J. Exp. Bot. 2021, No. erab413. 